\title{
Trends in suicide and unemployment in Scotland, 1976-86
}

\author{
I K Crombie
}

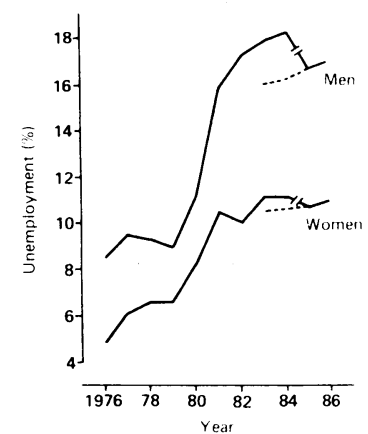

FIG 1-Trends in unemployment among men and women in Scotland, 1976-86

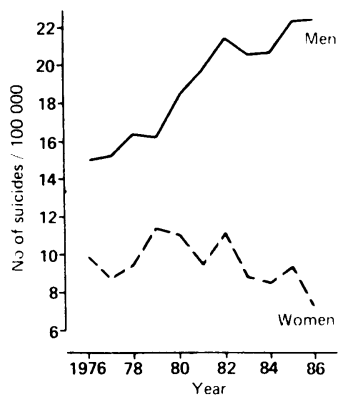

FIG 2-Trends in suicide of men and women aged $25-64,1976-86$

Department of Community

Medicine,

University of Dundee

Medical School, Ninewells

Hospital, Dundee

DD19SY

I K Crombie, PHD, senior

epidemiologist

\begin{abstract}
To establish whether a correlation exists between unemployment and suicide the trends in the rates of both among men in Scotland during 1976-86 were studied. Both rates showed rapid increases in the late 1970s and then much slower increases after 1982. In contrast, among women, although the rate of unemployment followed a similar pattern, there was a gradual fall in the rate of suicide. The trends in regional unemployment for men during 1971-81 were compared with the suicide rates for the period before the increase (1974-7) and the period after the rapid increase (1983-6). No association was observed between trends in suicide and unemployment when analysed by health board areas or aggregates of local government districts.
\end{abstract}

These data do not support the hypothesis that the rise in unemployment is a direct cause of the rise in suicide rates among men.

\section{Introduction}

Studies have shown that after a period of relative constancy in the early 1970s suicide rates among men rose considerably in the late $1970 \mathrm{~s}^{1.3}$ This rise took place against a background of increasing unemployment, and evidence for an association between suicide and unemployment is growing. ${ }^{+6}$ Whether the relation is directly causal at the individual level is, however, unclear, and there are inconsistencies in the association between unemployment and suicide nationally and regionally. ${ }^{+5}$ To determine the extent to which the recent increases in suicide rates were related to changes in unemployment I studied the national and regional trends in Scotland.

\section{Methods}

Unemployment data-National unemployment rates for 1976-86 were taken from three issues of the Unemployment Gazette (January 1982, January 1985, and February 1988). Regional data for 1981 were obtained from the census; those for 1971 were extracted from a magnetic tape of the 1971 census provided by the Registrar General (Scotland), in which the data had been recast to the geographical units of the reorganisation of local government in 1974 .

Suicide data-Details of each death from suicide (International Classification of Diseases codes E950-959) were extracted from a magnetic tape provided by the Registrar General (Scotland) as described elsewhere. ${ }^{3}$ The age range 25-64 was used for all analyses. This restricted age range was selected because it has shown a greater rate of increase in suicide ; older people were excluded because most of them are retired. Deaths were aggregated to local government districts and health board areas by using the postcode directory. Because of their small populations the three island health boards (Shetland, Orkney, and the Western Isles) were combined into a single unit.

Population data and mortality - Population data were obtained from the annual reports of the Registrar
General (Scotland) for the calendar years 1974-86. Mortality was expressed per 100000 , and the rates for a period of years used the average population for the data aggregated over that period.

Statistical methods-Spearman's rank correlation coefficient was used to assess the relation between unemployment and suicide.

\section{Results}

Figure 1 shows the trends in unemployment for men and women during 1976-86. Some care has to be taken in interpreting these trends because of changes in the definition of unemployment. This effect was minimised by obtaining data for which changes in definition occurred only in 1982 and 1985 . The first change resulted in a fall of about $0.5 \%$ in the unemployment rate and the second a fall of $2 \%$. When the equivalent data for 1983 and 1984 with the 1985 definition were studied the small upward trend during 1982-4 continued through to 1986. Three periods of increase were evident: a period of rapid rise during 1979-81, a period before this (1976-78.), and a period afterwards (1982-6).

Figure 2 shows the trends in suicide rates over the same period with data for men and women aged 25-64. For men there was a distinct increase in the rate, which began in the late 1970s and was largely over by 1982 . The starting point of the rapid increase cannot be precisely identified because of the year to year fluctuations in the rates. In contrast, the suicide rates for women showed a small overall fall over the period of rising unemployment. Similar analyses of the age ranges $15-54$ and 15 and over (data not shown) led to identical patterns.

The rank correlation between suicide and unemployment among men was $0.81(\mathrm{p}<0.01)$. To assess the consistency of the association the trends in suicide and unemployment in the 13 health board areas were examined. Published regional data on unemployment from the 1971 and 1981 censuses were used, and the increase in unemployment rates was compared with the increase in suicide from 1974-7 (the period before the rapid rise in unemployment) to $1983-6$ (the period after the rapid rise). Figure 3 shows no association between the increase in suicide and that in unemployment (rank correlation $r=0 \cdot 10$ ). Further analysis of the data from different periods showed that there were weak negative associations between the unemployment and suicide rates before the rapid rise; the rates of both after the rapid rise; and the suicide rates after the rapid rise with the rise in unemployment rates (1971-81) (table I).

Because regions are large and internally heterogeneous in terms of changes in unemployment, data were obtained for the 56 local government districts. Because they were too small individually to provide stable suicide rates they were aggregated into five groups on the basis of their unemployment rates in 1981; no association with suicide rate was observed (table II). These rates were based on comparatively large numbers of events and large populations, as evidenced by their small standard errors, so that the 
TABLE I - Association between suicide and unemployment rates in different periods

\begin{tabular}{|c|c|c|c|}
\hline \multicolumn{2}{|c|}{ Time period of data } & \multicolumn{2}{|c|}{ Rank correlation coefficient } \\
\hline Suicide & Unemployment & Men & Women \\
\hline 1974-7 (Before rapid rise ${ }^{\star}$ ) & 1971 & $-0 \cdot 18$ & -0.04 \\
\hline 1983-4 (After rapid rise ${ }^{\star}$ ) & 1981 & $-0 \cdot 28$ & $-0 \cdot 16$ \\
\hline $1983-6$ & 1971-81 & $-0 \cdot 24$ & $-0 \cdot 10$ \\
\hline Increase (before to after rapid rise ${ }^{\star}$ ) & $\begin{array}{c}\text { (Absolute increase) } \\
1971-81\end{array}$ & $0 \cdot 10$ & 0.06 \\
\hline & (Absolute increase) & & \\
\hline
\end{tabular}

${ }^{\star}$ Rapid rise occurred during 1979-81

TABLE II-Mean (SE) suicide rates/100000 men, 1983-6, for districts aggregated into fifths by their unemployment rates

\begin{tabular}{lccccc}
\hline & \multicolumn{5}{c}{ Ranked district aggregates* } \\
\cline { 2 - 6 } \multicolumn{1}{c}{$\begin{array}{c}\text { Time period of } \\
\text { unemployment }\end{array}$} & 1 & 2 & 3 & 4 & 5 \\
\hline 1981 & $20 \cdot 4(1.80)$ & $25 \cdot 5(2 \cdot 25)$ & $21 \cdot 4(1.33)$ & $22 \cdot 2(1.50)$ & $19 \cdot 8(1 \cdot 11)$ \\
Increase 1971-81 & $21 \cdot 0(1.67)$ & $21 \cdot 7(1.53)$ & $25 \cdot 0(1.82)$ & $19 \cdot 7(1.51)$ & $20 \cdot 5(1 \cdot 11)$ \\
\hline
\end{tabular}

*Unemployment rates were lowest in group 1 and highest in group 5.

absence of an increasing suicide rate with increasing unemployment rate was not due to sampling variation. A similar pattern was observed when aggregation was by the increase in unemployment during 1971-81. Both methods of aggregation showed that the highest grade of unemployment was related to the lowest or next to lowest suicide rate.

The impact of an increased unemployment rate might depend on the original rate. To investigate this the local government districts were divided into four groups according to initial unemployment (low or high) and whether they had a low or high increase in unemployment. The increase in suicide rates from 1974-7 to 1983-6 was of a similar magnitude in the four groups (table III). The group of districts with low unemployment in 1971 but that experienced a high increase in unemployment to 1981 had overall the largest increase in suicide, but this was not significantly different from the increases in the other groups.

TABLE III-Mean (SE) increase ${ }^{\star}$ in suicide rates/100000 men for districts grouped by initial unemployment rates (1971) and change in unemployment rate (1971-81) among men

\begin{tabular}{ccc}
\hline & \multicolumn{2}{c}{1971 Unemployment rate $(\%)$} \\
\cline { 2 - 3 } Increase in unemployment & $<5.7$ & $\geqslant 5.7$ \\
\hline$<41.81$ & $6 \cdot 6(1.87)$ & $5 \cdot 9(2.01)$ \\
$\geqslant 4.8$ & $9 \cdot 4(2.72)$ & $7 \cdot 0(1.21)$ \\
\hline
\end{tabular}

*Increase from 1974-7 to 1983-6.

\section{Discussion}

This study found a remarkable correlation between the trends in unemployment and suicide rates in men: the periods of a rapid increase in the rates and the subsequent slower increase coincided exactly. A simplistic interpretation would be that an increase in unemployment brings about an immediate increase in suicide. The hazards of imputing causation from correlation between trends over time in rates, however, are too well known for the observation to be considered other than interesting. The pattern is, however, consistent with that in the United States and with that in Britain when the confounding effect of the fall in the number of suicides by coal gas is removed.

The data for women contrast with those for men; although there was a rise in reported unemployment coincident with that for men, the suicide rates showed a small decrease. The interpretation of unemployment among women may be complicated by changes in the numbers of women registering as unemployed (as opposed to simply being unemployed). A detailed comparison of unemployment among men and women in Britain, however, concluded that women have experienced comparable increases in unemployment to men. ${ }^{x}$ The relation between unemployment and suicide in women is unclear. In Platt's review only five of the 30 studies that compared time trends specifically mentioned women. ${ }^{+}$One significant positive and one significant negative association between unemployment and suicide were found. Smith also emphasised that the association between suicide and unemployment is, at best, weak among women. ${ }^{5}$ The importance of comparing trends among men and women lies in what it could show about possible causal mechanisms. For example, if loss of social contact or poorer financial circumstances were the important factors then effects of unemployment on both men and women might be expected. If loss of self esteem was the important factor then men, with their traditional role of breadwinner, might be affected to a greater extent.

The size of the increase in the national suicide rate among men (about 50\% during 1976-86) suggests that a similar association with unemployment should be seen regionally. Although many previous studies reviewed by Platt failed to find consistent effects regionally, they used data from earlier periods, when possible associations would have been much weaker. ${ }^{+}$The analyses of recent data in this study, however, failed to show an effect both within health board areas and within the much smaller and more internally homogeneous local government districts. Moser et al provided suggestive evidence of an association in England and Wales (divided into three regions) during 1971-81. ${ }^{9}$ Another study found a weak non-significant association between the increase in long term unemployment from July 1980 to July 1981 and the trend in suicide rates during 1975-83. ${ }^{10}$ Overall the pattern was not as strong as might have been expected given the recent increase in suicide and unemployment rates.

The apparent lack of an association between unemployment and suicide regionally, is in contrast with the findings both nationally and individually. ${ }^{+}$The strongest evidence at the individual level comes from the Office of Population Censuses and Surveys' longitudinal study, which showed a twofold to threefold increase in suicide among the unemployed. ${ }^{112}$ Explanations for these conflicting findings might be regional differences in the meaning and impact of unemployment. The stated unemployment rates are net rates based on sizable flows in and out of employment $^{13}$ so that regions with similar net rates may have greatly different numbers of people unemployed. Furthermore, the impact of unemployment may depend on the chances of re-employment and on local attitudes to employment state. Finally, some types of unemployment, such as the closure of a local steel works or coal mine, may have a different impact from others, such as the loss of self employment or the closure of a small business. These factors might vary independently at a regional level, helping to conceal the association visible at the national level. An alternative explanation is that national unemployment rates are simply a marker for economic recession and some factor (or factors) associated with this influences suicide rates. ${ }^{+}$This other factor may not follow the same regional distribution as unemployment.

In conclusion, I found an association between trends in unemployment and suicide rates among men nationally but not regionally. These data do not support a rise in unemployment being a direct cause of the recent large increase in suicide among men.

I was supported by the Scottish Home and Health Department, and the data on suicide were obtained while I was at the cardiovascular epidemiology unit, Ninewells Hospital. I thank Professor C Florey for helpful comments on this manuscript. 
1 McLure GMG. Trends in suicide rate for England and Wales 1975-80. $\mathrm{Br} \mathcal{F}$ Psvchiatry 1984;144:119-26.

2 Bulusu L, Alderson M. Suicides 1950-82. Population Trends 1984;35:11-7.

Mcloone P, Crombie IK. Trends in suicide in Scotland, 1974-84: an increasing problem. Br.Med f 1987;295:629-31.

Platt S. Unemployment and suicidal behaviour: a review of the literature. Soc SciMed 1984;19:93-115

Smith R. "I couldn't stand it anymore": suicide and unemployment. Br Med $\mathcal{F}$ 1985;291:1563-6

6 Monk M. Epidemiologv of suicide. Epidemiol Rev 1987;9:51-70.

Anonymous. Unemployment adjusted for discontinuities and seasonality. Employment Gazette 1985:93:274-7.

8 Dex S, Perry SM. Woman's employment changes in the 1970's. Employment Gazetue 1984:92:151-64.

Moser KA, Fox AJ, Jones DR, Goldblatt PO. Unemployment and mortality: further evidence from the OPCS longitudinal study 1971-81. Lancel $1986 ; \mathrm{i}: 365-6$

10 Charlton JRH, Bauer R, Thakhore A, Silver R, Aristidou M. Unemployment and mortality: a small area analysis. I Epidemiol Community Health 1987;41:107-13.

11 Moser KA, Fox AJ, Jones DR. Unemployment and mortality in the OPCS longitudinal study. Lancet $1984 ;$;i: $1324-8$.

12 Moser KA, Goldblatt PO, Fox AJ, Jones DR. Unemployment and mortality: comparison of the 1971 and 1981 longitudinal study census samples. Br.Med F 1987;294:86-90.

13 Hughes PR, Hutchinson G. Changing characteristics of male unemployment flows, 1972-1981. Employment Gazette 1986;94:365-8.

(Accepted 10 fanuary 1989)
Foundation for Blood

Research, PO Box 190,

Scarborough, ME 04074, United States

Wendy Y Craig, PHD, research scientist

Glenn E Palomaki, BS, director, division of biometry James E Haddow, $\mathrm{MD}$, vice president

Correspondence to: $\mathrm{Dr}$ Haddow.

\title{
Cigarette smoking and serum lipid and lipoprotein concentrations: an analysis of published data
}

\author{
Wendy Y Craig, Glenn E Palomaki, James E Haddow
}

\section{Abstract}

To examine the association between cigarette smoking in adults and serum lipid and lipoprotein concentrations the results of 54 published studies were analysed. Overall, smokers had significantly higher serum concentrations of cholesterol $(3 \cdot 0 \%)$, triglycerides $(9 \cdot 1 \%)$, very low density lipoprotein cholesterol $(10.4 \%)$, and low density lipoprotein cholesterol $(1.7 \%)$ and lower serum concentrations of high density lipoprotein cholesterol $(-5 \cdot 7 \%)$ and apolipoprotein AI $(-4 \cdot 2 \%)$ compared with nonsmokers. Among non-smokers and light, moderate, and heavy smokers a significant dose response effect was present for cholesterol $(0,1 \cdot 8,4 \cdot 3$, and $4.5 \%$ respectively), triglycerides $(0,10 \cdot 7,11 \cdot 5$, and $18 \cdot 0 \%)$, very low density lipoprotein cholesterol $(0,7 \cdot 2,44 \cdot 4$, and $39.0 \%)$, low density lipoprotein cholesterol $(0$, $-1 \cdot 1,1 \cdot 4$, and $11 \cdot 0 \%$ ), high density lipoprotein cholesterol $(0,-4 \cdot 6,-6 \cdot 3$, and $-8 \cdot 9 \%)$, and apolipoprotein $A I(0,-3.7$ and $-5.7 \%$ in non-smokers and light and heavy smokers).

These dose response effects may provide new evidence for a causal relation between exposure to cigarette smoke and changes in serum lipid and lipoprotein concentrations whether as a direct result of physiological changes or of dietary changes induced by smoking. Adequate prospective data to estimate the excess risk of coronary artery disease existed only for cholesterol concentration. When that information was combined with data from the present study, and given that smokers as a group face an average overall excess risk of coronary artery disease of $70 \%$, it was estimated that the observed increased serum cholesterol concentration in smokers may account for at least $9 \%$ of that excess risk. Furthermore, the dose response effect of smoking on serum cholesterol concentration suggests a gradient of increased absolute risk of coronary artery disease between light and heavy smokers.

\section{Introduction}

Cigarette smokers have a higher risk of coronary artery disease than non-smokers. Several possible explanations have been offered for this association, including altered blood coagulation, ' impaired integrity of the arterial wall, ${ }^{2}$ and changes in blood lipid and lipoprotein concentrations. ${ }^{12}$ We examined the last of these explanations by analysing the extent to which published data support a relation between smoking and altered serum lipid and lipoprotein concentrations in adults. ${ }^{3.56}$ We then used information from this analysis to estimate the excess risk of coronary artery disease associated with smoking that may be accounted for by changes in serum cholesterol concentrations.

\section{Methods \\ DATA COLLECTION}

Articles relating to the effects of long term cigarette smoking on serum concentrations of cholesterol, triglycerides, very low density lipoprotein cholesterol, low density lipoprotein cholesterol, high density lipoprotein cholesterol, and apolipoprotein AI in adults were identified by using the Medline search facility (1966-87) and the database of the Office on Smoking and Health (United States Department of Health and Human Services, Rockville, Maryland). The reference lists of these articles were then reviewed to identify additional studies. Data from these sources were grouped into the following categories as defined by the original authors: non-smokers, all current smokers, and light, moderate, and heavy current smokers. In most studies lipid concentrations were not adjusted by the original authors for variations in age, sex, weight, alcohol consumption, or physical activity. Several, however, were adjusted for one or more of these variables. ${ }^{115242528303134+04952}$ Three of these studies provided both adjusted and unadjusted data, and there was no qualitative difference between the two. ${ }^{152425} \mathrm{~A}$ fourth study presented unadjusted data but reported that adjustment did not affect the results. ${ }^{55}$. For these reasons we did not make distinctions according to age, sex, or other population variables in the present analysis. When population subgroups other than groups exposed to cigarette smoke were reported we calculated a weighted mean for each available variable and used this value in the analysis.

Mass unit data were required for the present analysis so those studies in which the data were reported solely in the form of regression coefficients were excluded Eleven of the studies included in this analysis presented data in terms of mass units and as regression coefficients. Such data were available for all of the variables discussed in the present report with the exception of very low density lipoprotein cholesterol; in each case the conclusions drawn did not differ between the two methods of analysis. We also excluded studies that contained insufficient data for analysis, such as those that omitted the number of subjects in each category of smoking and those that investigated ex-smokers, pipe or cigar smokers, or the short term effects of smoking. 